\title{
Laminar-turbulent reactive flows in porous media
}

\section{Andrea Corli* and Haitao Fan}

\begin{abstract}
We state some recent results concerning liquid-vapor phase transitions for a fluid flow through a porous medium. The focus is on the friction exerted by the porous medium, which is modeled in such a way to include both laminar and turbulent flows. In this way we obtain a hyperbolic system of three balance laws with a forcing term that is discontinuous in the state variables. Existence, uniqueness and qualitative behavior of traveling waves is proved by a novel regularization technique.
\end{abstract}

Keywords: Riemann problem, phase transitions, hyperbolic conservation laws.

Mathematical subject classification: 35L65, 35L67, 76T30.

\section{Introduction}

In this paper we provide some recent results about the existence and qualitative behavior of traveling waves for a hyperbolic model of fluid flow through a porous medium. We make the strong assumption that the flow is isothermal and inviscid; this simplification allows us to focus on two features of the flow. On the one hand, we model the occurrence of laminar and turbulent flows, by means of a source term in the momentum equation that is discontinuous as a function of the state variables; on the other hand, we let the fluid undergo liquid-vapor phase transitions. Such results extend to the case of laminar-turbulent flows the analysis that we began in $[5,6]$.

Received 27 March 2015.

*Corresponding author. 
Our model is the following:

$$
\left\{\begin{array}{l}
v_{t}-u_{x}=0, \\
u_{t}+p(v, \lambda)_{x}=-\alpha(u) u, \\
\lambda_{t}=\frac{1}{\tau}\left(p(v, \lambda)-p_{e}\right) \lambda(\lambda-1),
\end{array}\right.
$$

for $t>0$ and $x \in \mathbb{R}$. Above, we denoted by $v>0$ the specific volume, $u$ the velocity, $p$ the pressure, $\lambda \in[0,1]$ the mass-density fraction of the vapor in the fluid. The constants $\tau>0$ and $p_{e}>0$ represent a characteristic reaction time and the equilibrium pressure, respectively. The pressure $p(v, \lambda)$ is assumed to be smooth and satisfy

$$
p_{v}<0, \quad p_{\lambda}>0, \quad p_{v v}>0, \quad p_{v \lambda}<0 .
$$

The focus of this research is about the effects on the flow due to the coefficient $\alpha(u)>0$, which takes into account the friction exerted onto the fluid by the porous media; the case of a flow in a rough pipe is completely analogous. For general information on the physical features of this subject we refer to [16].

When the flow speed is sufficiently slow, then $\alpha$ is constant and the flow is called laminar. For high flow speeds, on the contrary, $\alpha$ does depend on $u$ and indeed increases with $u$; in this case the flow is called turbulent. In the latter case we assume, as usual in the applied literature, that $\alpha$ is linear but different growths can be considered as well. Between the laminar and the turbulent regime there is a small intermediate zone of flow speeds where, however, there is no reliable value for $\alpha$. In our model we simply admit that this transition zone is reduced to a single value $u^{*}$, and across it $\alpha$ has a jump. More precisely we assume

$$
\alpha(u)= \begin{cases}a & \text { if }|u|<u^{*} \\ b|u| & \text { if }|u| \geq u^{*}\end{cases}
$$

for some $a>0, b>0, u^{*}>0$ and $a<b u^{*}[13,14,16]$. Thus, this paper fall into the framework of balance systems whose source term is discontinuous in the state variables; we refer to $[1,9,10,11,12]$ for other models and results on this topic.

The study of traveling waves in the case $\alpha=0$ was done in [8]. If $\alpha$ is a strictly positive constant, then traveling waves for system (1.1) were constructed in [5] for a large range of significant end states. These results were extended to the case when $\alpha$ depends on $\lambda$ but is bounded away from zero in [3]. The degenerate situation where $\alpha(\lambda)>0$ for $\lambda \in[0,1)$ but $\alpha(1)=0$ was considered in [6]. On 
the contrary, in this paper we focus on the case where $\alpha$ depends upon $u$; the general case where $\alpha$ depends both on $\lambda$ and on $u$ can be treated at the only price of greater technical difficulties.

Our mathematical approach in treating system (1.1) is inspired by the physical modeling of the laminar-turbulent transition. In order to let traveling waves cross the discontinuity at $u^{*}$, we regularize the friction coefficient $\alpha(u)$ in an small interval of width $\epsilon>0$ around $u^{*}$. Once we prove the existence of $\epsilon$ dependent traveling waves, we pass to the limit for $\epsilon \rightarrow 0$. An interesting result that we obtain is that the limit solution, which has to be meant in the sense of differential inclusions, does not depend on the regularization if the regularization is monotone-preserving. Roughly speaking, this says that the modeling of $\alpha(u)$ in the real $\epsilon$-wide transition zone plays no role in the problem.

Section 2 below contains the statement of the main result. In Section 3 we give a very brief sketch of the proof and add further comments. Complete proofs are provided in [4].

\section{Main Result}

A traveling wave $(v, u, \lambda)$ to (1.1) with constant speed $c$ is a solution to (1.1) of the form $(v, u, \lambda)(x, t)=U\left(\frac{x-c t}{\tau}\right)$, where $U=U(\xi)$ is defined in $\mathbb{R}$ and valued in $\mathbb{R}^{3}$. We can assume without any loss of generality that $c \geq 0$. To find out unique solutions we impose conditions on $U$ at $\xi= \pm \infty$; this lead to consider the following problem:

$$
\left\{\begin{array}{l}
-c v^{\prime}-u^{\prime}=0, \\
-c u^{\prime}+p^{\prime}=-A(u) u, \\
-c \lambda^{\prime}=\left(p-p_{e}\right) \lambda(\lambda-1), \\
(v, u, \lambda)( \pm \infty)=\left(v_{ \pm}, u_{ \pm}, \lambda_{ \pm}\right), \\
\left(v^{\prime}, u^{\prime}, \lambda^{\prime}\right)( \pm \infty)=0 .
\end{array}\right.
$$

We denoted $A(u)=\alpha(u) \tau$. A necessary condition for solving (2.1) is that the end states are equilibria for the dynamical system, i.e., both conditions

$$
u_{ \pm}=0 \quad \text { and } \quad\left(p_{ \pm}-p_{e}\right) \lambda_{ \pm}\left(\lambda_{ \pm}-1\right)=0
$$

must be satisfied. By (2.1) we deduce a further necessary condition involving $c$ :

$$
c\left(v_{+}-v_{-}\right)=0 .
$$


Then, either $c=0$ or

$$
c>0 \quad \text { and } \quad v_{-}=v_{+}=: \bar{v} .
$$

The former case admits only trivial solutions, see [5], and then we focus on (2.3). Since by the first equation in (2.1) it follows that $u=-c(v-\bar{v})$, we can define $B(v)=A(-c(v-\bar{v}))$; if we denote $v_{ \pm}^{*}:=\bar{v} \pm \frac{u^{*}}{c}$, we see that the term $B$ is given by

$$
B(v)= \begin{cases}\tau a & \text { if } v \in\left(v_{-}^{*}, v_{+}^{*}\right), \\ \tau b c|v-\bar{v}| & \text { if } v \in\left(0, v_{-}^{*}\right] \cup\left[v_{+}^{*}, \infty\right) .\end{cases}
$$

Therefore we can rewrite (2.1) as

$$
\left\{\begin{array}{l}
c s v^{\prime}=g \\
\lambda^{\prime}=-\frac{1}{c}\left(p-p_{e}\right) \lambda(\lambda-1) \\
(v, \lambda)( \pm \infty)=\left(\bar{v}, \lambda_{ \pm}\right)
\end{array}\right.
$$

for $(\lambda, v) \in \Omega:=[0,1] \times(0, \infty)$, where

$$
s(\lambda, v)=c^{2}+p_{v}, \quad g(\lambda, v)=B c^{2}(v-\bar{v})+p_{\lambda}\left(p-p_{e}\right) \lambda(\lambda-1) .
$$

We point out that the function $g$ is discontinuous along the lines $v=v_{ \pm}^{*}$ with $v_{ \pm}^{*}>0$.

Now, we introduce the following subsets of $\Omega$ :

$$
S=\{s(\lambda, v)=0\}, \quad \mathcal{P}=\left\{p(v, \lambda)=p_{e}\right\}, \quad G:=\{g(\lambda, v)>0\} .
$$

By (1.2), the sets $S$ and $\mathcal{P}$ are easily shown to be graphs of two smooth functions $v^{\mathcal{S}}(\lambda)$ and $v^{\mathcal{P}}(\lambda)$, respectively. The boundary $G$ of $G$ can be written as the union of two subsets, namely, $G=G_{0} \cup G_{\text {disc }}$, where $\mathcal{G}_{0}=\{g(\lambda, v)=0\}$ is the null set of $g$ and

$$
G_{d i s c}=\left\{v=v_{ \pm}^{*}, g(\lambda, v+) \cdot g(\lambda, v-) \leq 0\right\} .
$$

About $G$, we notice that $g(0, v)=0$ or $g(1, v)=0$ if and only if $v=\bar{v}$. Moreover, $g(0, v)>0$ if $v>\bar{v}$ and $g(0, v)<0$ if $v<\bar{v}$; the same holds for $g(1, v)$. Since the function $\lambda \mapsto g(\lambda, v)$ is smooth for every $v \in(0, \infty)$, it follows that

$$
G_{\text {disc }} \subset\left\{\delta<\lambda<1-\delta, v=v_{ \pm}^{*}\right\},
$$

for some $\delta>0$. As in [6], we write for short "below"("above") for "strictly below"(resp., "strictly above"). Moreover, if $\mathcal{A}$ and $\mathcal{B}$ are two sets in the 
$(\lambda, v)$-plane we write $\mathcal{A} \prec \mathcal{B}(\mathcal{A} \succ \mathcal{B})$ to mean that $\mathcal{A}$ lies below (resp., above) $\mathcal{B}$.

The following result provides necessary conditions for the existence of traveling waves.

Lemma 2.1 ([5]). Traveling wave to (2.4) exist only if the end states $\left(\bar{v}, \lambda_{ \pm}\right)$ satisfy one of the following conditions:

(i) $p_{ \pm} \geq p_{e}$ and $\lambda_{-}=0, \lambda_{+}=1$;

(ii) $p_{ \pm} \leq p_{e}$ and $\lambda_{-}=1, \lambda_{+}=0$;

(iii) $p_{+}>p_{e}=p_{-}$and $\lambda_{-}<1, \lambda_{+}=1$;

(iv) $p_{+}<p_{e}=p_{-}$and $\lambda_{-}>0, \lambda_{+}=0$;

(v) $p_{-}=p_{+}=p_{e}$ and $\lambda_{-}=\lambda_{+}$.

The main difficulty in the study of the traveling waves for (1.1) is that, owing to the discontinuities of $g$, the trajectories of (2.4) stop when they intersect the lines $v=v_{ \pm}^{*}$ at points which do no lye on $S$. For this reason we regularize $B$ (and consequently $g$ ) in the region

$$
\mathcal{R}_{\epsilon}=\left\{(\lambda, v) \in \Omega:\left|v-v_{ \pm}^{*}\right|<\epsilon\right\},
$$

for a small number $\epsilon>0$, see Figure 1; there, we denoted $\Omega_{\text {lam }}=\{v \in$ $\left.\left(v_{-}^{*}, v_{+}^{*}\right)\right\}$ and $\Omega_{\text {turb }}=\left\{v \in\left(0, v_{-}^{*}\right] \cup\left[v_{+}^{*}, \infty\right)\right\}$. We denote by $B_{\epsilon}$ and $g_{\epsilon}$ the smoothed functions.

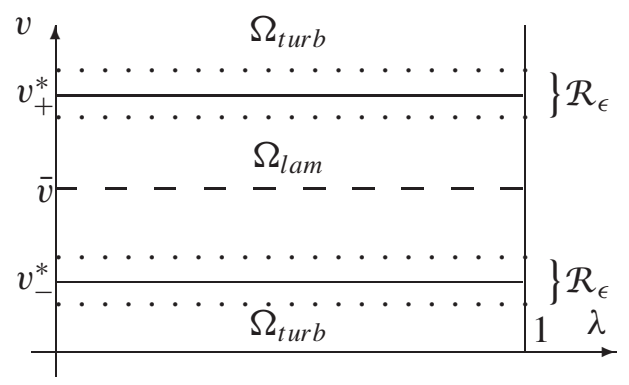

Figure 1: The smoothing region $\mathcal{R}_{\epsilon}$.

In the following we require that the smoothing is monotone-preserving:

$$
\begin{array}{r}
B_{\epsilon}(v) \text { is increasing (decreasing) where } B(v) \text { is increasing } \\
\text { (resp., decreasing). }
\end{array}
$$


Then, we are led to considering the regularized problem

$$
\left\{\begin{array}{l}
c s v^{\prime}=g_{\epsilon} \\
\lambda^{\prime}=-\frac{1}{c}\left(p-p_{e}\right) \lambda(\lambda-1) \\
(v, \lambda)( \pm \infty)=\left(\bar{v}, \lambda_{ \pm}\right)
\end{array}\right.
$$

We say that $(v, \lambda)(\xi)$ is a traveling-wave solution of (1.1) in the sense of the vanishing smoothing if it is obtained as the uniform limit of solutions $\left(v_{\epsilon}, \lambda_{\epsilon}\right)(\xi)$ of $(2.5)_{\epsilon}$ for $\epsilon \rightarrow 0$. Clearly, such a limit solution can satisfy (1.1) only in the sense of differential inclusions because of the discontinuities of $g$.

Here follows our main result.

Theorem 2.2. Assume (1.2), (1.3), (2.3), (2.5), consider end states with $u_{ \pm}=0$. Assume that one of the following conditions is satisfied:

(i) $p_{ \pm} \geq p_{e}, \lambda_{-}=0, \lambda_{+}=1$; moreover $G$ and $S$ intersect at most once with a transverse intersection.

(ii) $p_{ \pm} \leq p_{e}, \lambda_{-}=1, \lambda_{+}=0$; moreover, $G \cap S=\emptyset$ and $B c^{2}+$ $\left(p_{\lambda}\left(p-p_{e}\right)\right)_{v} \lambda(\lambda-1)>0$ for $v$ near $v_{-}^{*}$.

(iii) $p_{+}>p_{e}=p_{-}, \lambda_{-}<1, \lambda_{+}=1$; moreover $\mathcal{G}$ and $S$ intersect at most once in the region $\left(\lambda_{-}, 1\right] \times \mathbb{R}$ with a transverse intersection.

(iv) $p_{+}<p_{e}=p_{-}, \lambda_{-}>0, \lambda_{+}=0$ and $G \cap S=\emptyset$.

(v) $p_{-}=p_{+}=p_{e}$ and $\lambda_{-}=\lambda_{+}$.

Then, there is a unique, up to a shift in $\xi$, traveling-wave solution of (1.1) in the sense of the vanishing smoothing; in case (v) the solution is constant.

Moreover, assume on the pressure the further conditions

$$
\lim _{v \rightarrow 0+} p(v, \lambda)=+\infty, \quad \lim _{v \rightarrow+\infty} p(v, \lambda)=0,
$$

for every $\lambda \in[0,1]$. If the end states satisfy $u_{ \pm}=0$ and one of the necessary conditions in Lemma 2.1, then there is a unique (up to a shift in $\xi$ ) traveling-wave solution to (1.1) if $c>0$ is either sufficiently large or sufficiently small.

We emphasize that the solution $(v, \lambda)(\xi)$ obtained by the vanishing smoothing in the above theorem satisfies the end-state conditions $(v, \lambda)( \pm \infty)=\left(\bar{v}, \lambda_{ \pm}\right)$ and equation $(2.4)_{2}$ for every $\xi$; equation (2.4) is satisfied for every $\xi$ such that 
$v(\xi) \neq v_{ \pm}^{*}$ while when $v(\xi)=v_{ \pm}^{*}$ a differential inclusion for $v^{\prime}$ holds. In the case of condition (i) in the above theorem, for instance, this condition can be written as

$$
v^{\prime}(\xi) \in\left[\frac{g\left(\lambda(\xi), v_{+}^{*}+\right)}{c s\left(\lambda(\xi), v_{+}^{*}+\right)}, \frac{g\left(\lambda(\xi), v_{+}^{*}-\right)}{c s\left(\lambda(\xi), v_{+}^{*}\right)}\right] .
$$

\section{A sketch of the proof and further comments}

We only consider Case (i) and focus on the subcase when

$$
G_{0} \cap \Omega_{\text {lam }} \neq \emptyset, \quad G_{0} \cap \Omega_{\text {turb }} \neq \emptyset \quad \text { and } \mathcal{P} \nprec\left\{v=v_{+}^{*}\right\} .
$$

Indeed, it is not difficult to see that all the other subcases can be solved by the same techniques of [5]. Under the assumption (3.1), the sets $\mathcal{G}_{0} \cap \Omega_{\text {lam }}$ and $G_{0} \cap \Omega_{\text {turb }}$ are connected by segments lying on the line $\left\{v=v_{+}^{*}\right\}$; in turn, the set $G_{d i s c}$ is the union of these segments. The sets $G$ and $G_{\epsilon}$ only differ in the region

$$
\mathcal{N}_{\epsilon}:=\left\{(\lambda, v) \in \mathcal{R}_{\epsilon}:\{\lambda\} \times\left(v_{+}^{*}-\epsilon, v_{+}^{*}+\epsilon\right) \cap G \neq \emptyset\right\} .
$$

and we may assume without loss of generality $\mathcal{N}_{\epsilon}$ is composed by two segments:

$$
\mathcal{N}_{\epsilon}=\mathcal{N}_{\epsilon, 1} \cup \mathcal{N}_{\epsilon, 2},
$$

where $\mathcal{N}_{\epsilon, 1}=\left[v_{1}, v_{2}\right] \times\left(v_{+}^{*}-\epsilon, v_{+}^{*}+\epsilon\right)$ and $\mathcal{N}_{\epsilon, 2}=\left[v_{3}, v_{4}\right] \times\left(v_{+}^{*}-\epsilon, v_{+}^{*}+\epsilon\right)$. We also denote $\mathcal{L}_{\epsilon}=\left(v_{2}, v_{3}\right) \times\left(v_{+}^{*}-\epsilon, v_{+}^{*}+\epsilon\right)$.

Assume moreover that $\mathcal{G} \prec S$, see Figure 2, and then that $G_{\epsilon} \prec S$, assuming $\epsilon$ sufficiently small. The existence and uniqueness of a solution to $(2.5)_{\epsilon}$ can be proved by a suitable modification of arguments already used in [5]. The crucial step in the proof consists in showing that the family $\left\{\left(v_{\epsilon}, \lambda_{\epsilon}\right)(\xi)\right\}$ is Cauchy for $\epsilon>0$. If we denote by $W_{\epsilon_{1}}(\lambda):=\left(\lambda, v_{\epsilon_{1}}(\lambda)\right)$ and $W_{\epsilon_{2}}(\lambda):=\left(\lambda, v_{\epsilon_{2}}(\lambda)\right)$ the trajectories of two solutions, this is done by carefully examining the behavior of $W_{\epsilon_{1}}$ and $W_{\epsilon_{2}}$ in the phase plane $(\lambda, v)$ when they enter or exit the regions $\mathcal{N}_{\epsilon}$ or $\mathcal{L}_{\epsilon}$, for $\epsilon:=\max \left\{\epsilon_{1}, \epsilon_{2}\right\}$. Once that the Cauchy property has been proved, the existence of the limit for $\epsilon \rightarrow 0$ in the $C([0,1])$ norm follows. Also proving that the limit $(v, \lambda)(\xi)$ is a solution gives no additional difficulties.

The case when $S \prec G$ is studied in an analogous way. The last case to be considered is when $S \cap G=\left(\lambda_{0}, v_{0}\right)$, see Figure 3. Since $S$ and $\mathcal{G}$ intersect transversely, also the sets $S$ and $\mathcal{G}_{\epsilon}$ intersect once and we denote by $\left(\lambda_{0}^{\epsilon}, v_{0}^{\epsilon}\right)$ their point of intersection. Remark that system $(2.5)_{\epsilon}$ is highly singular at $\left(\lambda_{0}^{\epsilon}, v_{0}^{\epsilon}\right)$ because both $s$ and $g_{\epsilon}$ in the first equation vanish. The existence proof requires 


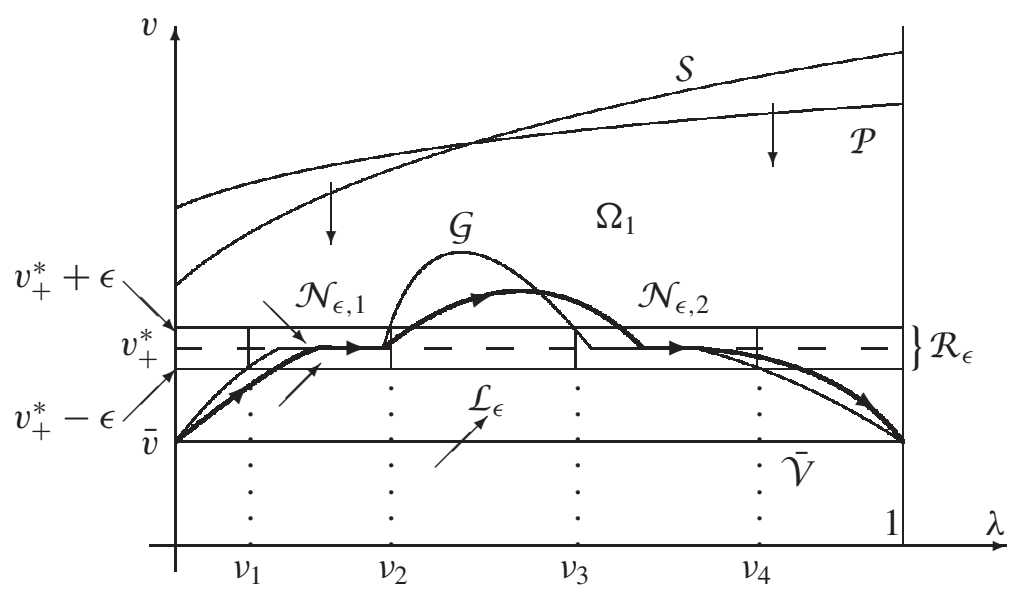

Figure 2: Case (i), $S \succ G$. The thick line is the limit trajectory of (2.4); arrows denote field directions.

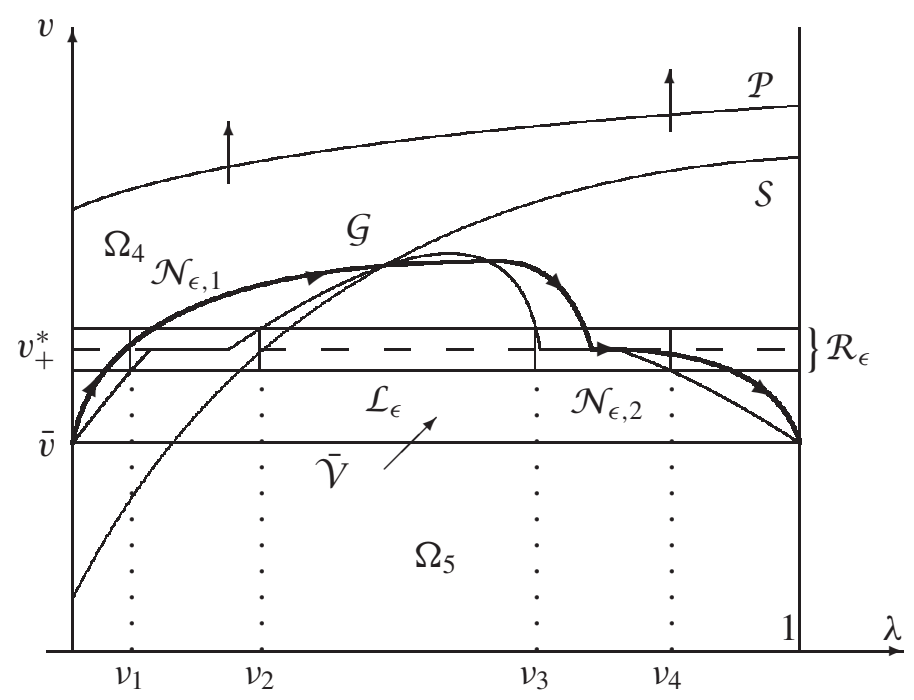

Figure 3: Case (i), $S \cap G \neq\{\emptyset\}$.

some subtle arguments that have already been exploited in [5] while the rest of the proof follows the lines of the previous case.

We point out that the double degeneracy encountered in the case $S \cap G=$ $\left(\lambda_{0}, v_{0}\right)$ occurs also in other papers. In the paper [15], the curve $S$ is called the wall of singularities and the intersection point $S \cap G$ the hole in the wall: in a naive way, it is through the hole that the trajectory can pass beyond the wall. An analogous example occurs in modeling the spreading of pollution $[2,7]$. 


\section{References}

[1] D. Amadori, L. Gosse and G. Guerra. Godunov-type approximation for a general resonant balance law with large data. J. Differential Equations, 198(2) (2004), 233-274.

[2] E. Barbera, C. Currò and G. Valenti. A hyperbolic model for the effects of urbanization on air pollution. Appl. Math. Model., 34(8) (2010), 2192-2202.

[3] A. Corli and H. Fan. A hyperbolic model for phase transitions in porous media. In: F. Ancona, A. Bressan, P. Marcati, and A. Marson (Eds). Hyperbolic Problems: Theory, Numerics, Applications. Amer. Inst. Math. Sci., (2014), 475-482.

[4] A. Corli and H. Fan. Phase transitions for laminar-turbulent flows in a pipeline or through porous media. Commun. Inf. Syst., 13(2) (2013), 151-181.

[5] A. Corli and H. Fan. Traveling waves of phase transitions in porous media. Appl. Anal., 92 (2013), 1217-1240.

[6] A. Corli and H. Fan. Traveling waves in porous media with phase-dependent damping. Nonlinear Anal. Real World Appl., 19 (2014), 135-149.

[7] C. Currò and D. Fusco. Discontinuous travelling wave solutions for a class of dissipative hyperbolic models. Atti Accad. Naz. Lincei Cl. Sci. Fis. Mat. Natur. Rend. Lincei (9) Mat. Appl., 16(1) (2005), 61-71.

[8] H. Fan. Traveling waves, Riemann problems and computations of a model of the dynamics of liquid/vapor phase transitions. J. Differential Equations, 150(2) (1998), 385-437.

[9] G. Guerra. Well-posedness for a scalar conservation law with singular nonconservative source. J. Differential Equations, 206(2) (2004), 438-469.

[10] J. Hong and B. Temple. The generic solution of the Riemann problem in a neighborhood of a point of resonance for systems of nonlinear balance laws. Methods Appl. Anal., 10(2) (2003), 279-294.

[11] J. Hong and B. Temple. A bound on the total variation of the conserved quantities for solutions of a general resonant nonlinear balance law. SIAM J. Appl. Math., 64(3) (2004), 819-857.

[12] E. Isaacson and B. Temple. Convergence of the $2 \times 2$ Godunov methodfor a general resonant nonlinear balance law. SIAM J. Appl. Math., 55(3) (1995), 625-640.

[13] M. Luskin. On the existence of global smooth solutions for a model equation for fluid flow in a pipe. J. Math. Anal. Appl., 84(2) (1981), 614-630.

[14] M. Luskin and B. Temple. The existence of a global weak solution to the nonlinear waterhammer problem. Comm. Pure Appl. Math., 35(5) (1982), 697-735.

[15] G. Pettet, D. McElwain and J. Norbury. Lotka-volterra equations with chemotaxis; walls, barriers and travelling waves. IMA J. Math. Appl. Med. Biol., 17 (2000), 395-413.

[16] F.M. White. Fluid Mechanics. McGraw-Hill, Boston, fourth edition (2003). 


\section{Andrea Corli}

Department of Mathematics and Computer Science

University of Ferrara

44121 Ferrara

ITALY

E-mail: crl@unife.it

\section{Haitao Fan}

Department of Mathematics

Georgetown University

Washington, DC 20057

USA

E-mail: fanh@georgetown.edu 